\title{
Joint Modeling of Longitudinal Pulse Rate and Time-to-Default from Treatment of Congestive Heart Failure Patients
}

\author{
Yikeber Abebaw (D) \\ Kasim Mohammed ${ }^{2}$ \\ Adem Aragaw iD ${ }^{3}$ \\ Bezanesh Melese' \\ 'Department of Statistics, Debre Tabor \\ University, Debre Tabor, Ethiopia; \\ ${ }^{2}$ Department of Statistics, University of \\ Gondar, Gondar, Ethiopia; ${ }^{3}$ Department \\ of Statistics, Mizan Tepi University, Mizan \\ Tepi, Ethiopia
}

Background: Globally, heart failure is a rapidly growing public health problem with an estimated prevalence of $>37.7$ million. It is a shared chronic phase of cardiac functional loss secondary to many etiologies.

Objective: The main purpose of this study was to investigate the risk of longitudinal change in pulse rate on time to default from treatment among congestive heart failure patients.

Methods: Hospital-based retrospective studies were conducted among 302 congestive heart failure patients who were 15 years old or older and who were on treatment follow-up from the 1 February 2016 to 31 December 2018 in Felege-Hiwot Referral Hospital, Bahir Dar, Ethiopia. Data were entered using SPSS version 23 and analyzed using SAS and R software. First, data were analyzed using linear mixed model and survival models separately, and then the joint models of both sub-models were analyzed using joint model analysis.

Results: Out of the total of 302 respondents, $34.1 \%$ of the respondents defaulted from treatment. About 55.2\% male respondents are defaulting and the remaining were censored. The results for separate and joint models were quite similar to each other but not identical. However, the estimated association parameter $(\alpha)$ in the joint model is $(\mathrm{HR}=1.0311,95 \%$ CI: $1.0033,1.0597, \mathrm{P}=0.0278)$, providing there is evidence of a positive significant relationship between the survival and the longitudinal sub-models. Thus, defaulting is more likely to occur in patients with higher pulse rates. A patient, who are male, New York Heart Association class IV, had low left ventricular ejection fraction and comorbid with hypertensive, chronic kidney disease, pneumonia were risk factors of pulse rate change and defaulting from treatment of congestive heart failure patients.

Conclusion: The joint model was preferred for simultaneous analyses of repeated measurement and survival data. Thus, the longitudinal measure pulse rate had a positive significant effect on time to default from treatment of patients.

Keywords: pulse rate, congestive heart failure, time to default, survival analysis, longitudinal analysis, joint model

\section{Introduction}

Globally, heart failure is a rapidly growing public health issue with an estimated prevalence of $>37.7$ million. ${ }^{1}$ It is a shared chronic phase of cardiac functional loss secondary to many etiologies. In the USA, the total medical costs for patients with heart failure are expected to rise from US\$20.9 billion in 2012 to $\$ 53.1$ billion by 2030. ${ }^{1}$ Over 1 million individuals within the UK have heart failure, with prevalence rates expected to extend over the subsequent decade. This places a big financial burden on the United Kingdom. ${ }^{2}$
Correspondence: Yikeber Abebaw Tel +25I 918558095

Email ykebera@gmail.com 
In Africa, $7 \%$ to $10 \%$ of all medical admissions to the hospital had cardiac diseases, out of this $3 \%$ to $7 \%$ had heart failure. Compared with the developed world Africa has the highest proportion of heart failure patients. In Africa it has a greater effect on the health and health economics. $^{3}$

In Sub-Saharan Africa (SSA), heart failure is the main public health concern and is associated with high morbidity and mortality, high rates of recurrent hospitalization, poor quality of life, and loss of economic productivity, as it affects mostly young and economically active adults. However, due to economic limitations, it is difficult and challenging for most heart failure patients in SSA to get the essential treatment. They have difficulty preserving regular follow-up visits and as a result cumulative premature deaths have occurred. ${ }^{4}$

In Ethiopia, approximately $9 \%$ of all deaths were caused by heart failure according to a $\mathrm{WHO}^{5}$ report. Heart failure has a cumulative problem, especially in urban settings in Ethiopia. It is one of the prevalent causes of morbidity ranging from $4 \%$ to $24 \%$, and mortality ranging from $6.5 \%$ to $24 \%$. It is highly prevalent among the older population, and the leading cause of medical intensive care unit admission ranging from $8.9 \%$ to 9.8\%. In Addis Ababa, an average of $25 \%$ of all household deaths, and $11 \%$ of all hospital deaths were due to heart failure. ${ }^{5}$ In Felege-Hiwot Referral Hospital (FHRH) heart failure is a progressive public health problem with high morbidity and mortality. ${ }^{6}$

The heart rate/pulse rate is the number of times the heart beats in one minute and it is used to know whether there is an abnormal or irregular pulse rate, or a heart block occurred or not. The normal resting heart rate is nearly 60 to 80 beats per minute. ${ }^{7}$ There is no common body part of a patient for taking a heart rate measurement. ${ }^{8}$ It can be measured in different parts of the body, but the two most common parts of the body are the wrist and the neck. ${ }^{9}$ Epidemiological and randomized clinical trial studies are focused on the predictive value of the heart rate. It proposes that a continued increment in heart rate increases the progression as well as the severity of the disease. ${ }^{10}$ A greater heart rate measure is associated with a high risk of congestive heart failure (CHF) hospitalization and an inferior pulse rate measure at the base line and through follow-up time is positively related with minor risk of mortality and re-hospitalization. ${ }^{11}$

Although the advancement in therapy and treatment of CHF is high, nowadays it is a chronic illness with an unpromising diagnosis, and has a high socioeconomic problem. ${ }^{12} \mathrm{CHF}$ is one of the major causes of hospitalization and high rates of mortality in several countries. ${ }^{13}$ Even though the number of CHF patients admitted to CHF clinics is increasing from year to year, there are a number of CHF related deaths and losses to follow-ups every year. This indicates that there are other factors affecting the progression of the disease and the survival status of CHF patients as well. Therefore, it is essential to assess risk factors associated with the effectiveness of the treatment over time. Most researchers conducted their study in relation to congestive heart failure patients using cross-sectional study, survival time of the patients after they were admitted to CHF clinic, and linear mixed models. However, Seid et $\mathrm{al}^{14}$ suggests that these linear mixed effect model analysis for longitudinal data, and Weibull or semi-parametric (Cox) proportional hazard model analysis for survival data separately were inappropriate when the longitudinal variable is correlated with patient health status (survival time), because of the survival endpoint occurrences, as well as the possibility of study dropout. Moreover, Grover et $\mathrm{al}^{15}$ studied the joint modeling of both longitudinal and survival data analysis, containing all information simultaneously, and gives lawful and effective inferences. Therefore, it is usually recommended to jointly model the longitudinal data analysis and survival data analysis altogether via a shared random effects model in order to account for the dependence between longitudinal and survival components on the same subject and any available covariates.

To the best of our knowledge, there is no research conducted on joint model analysis of longitudinal pulse rate and survival end time to default from CHF treatment and no research conducted on survival analysis of time to default from treatment of CHF patients. However, defaulting remains a public health problem, which we need to address. A person who was defaulting from congestive heart failure treatment is at risk for clinical worsening and difficulties including deterioration in drug opposition and death. Hence, we mainly aimed to investigate the risk of longitudinal change in pulse rate on time to default among congestive heart failure patients from 1st February 2016 to 31th December 2018 at Felege-Hiwot Referral Hospital. Specifically we aimed, i) to estimate the effect of baseline covariates on repeated measure pulse rate and time to default from treatment jointly, ii) to observe the association between longitudinal measure pulse rate and time to default from treatment among 
congestive heart failure patients, and iii) to demonstrate the advantage of joint model analysis techniques over separate survival and longitudinal analysis.

\section{Materials and Methods Study Design and Area}

This study was carried out by using a retrospective cohort study design based on data obtained from Felege-Hiwot Referral Hospital, Bahir-Dar, Amhara, Ethiopia. The Amhara Regional state is located in the northwestern part of Ethiopia. It is located $563 \mathrm{~km}$ from Addis Ababa, the capital city of Ethiopia. Felege-Hiwot Referral Hospital is a teaching and referral hospital and one of the oldest hospitals in Ethiopia. It is a tertiary health care labeled hospital helping the people of Bahir Dar town and distant area populations as a teaching and referral hospital; delivering health services to their dependents (patients), as well as public patients referred by other hospitals.

\section{Study Period and Source of Data}

In this study, we used secondary sources of data. The data had been obtained from the Amhara region at FHRH Outpatient Department in the CHF clinic during the follow-up time of 1st February 2016 to 31 th December 2018. The data that we used for this study was extracted from the chart of all CHF patients who were under follow-up. After we had given sufficient orientation to them about the aim of the study, way of data collection, and the variables that were included in the study the health professionals collected the data.

\section{Study Population}

Patients aged 15 years old and above, attended at least for 8 months (had at least two follow-up visits) for refilling their treatment, and who started the treatment during the study period at FHRH were involved in the study. Therefore, among the total $1334 \mathrm{CHF}$ patients registered from 1st February 2016 to 31th December 2018, only 302 $\mathrm{CHF}$ patients satisfied the inclusion criteria and hence are included in this study.

\section{Study Variable}

The two response variables, the longitudinal measured pulse rate - number of heart beats per minute measured every 4 months irrespective of their visit to $\mathrm{CHF}$ patient clinic, and the survival outcome - time to default from treatment for CHF patients were considered for this study. Defaulters used in this study were those patients who missed contact for at least eight months (who had missed two or more clinical appointments), which may be due to death, loss to follow-up, or transferring to another hospital.

Explanatory variables: Covariates associated with repeated measure pulse rate and time to default were gender (female, male), residence (rural, urban), age, type of $\mathrm{CHF}$ (left side failure, right side failure, biventricular failure), etiology of heart failure (valvular heart disease (VHD), hypertensive heart disease(HHD), ischemic heart disease (IHD), cor pulmonale, dilated cardiomyopathy, other etiologies), left ventricle ejection fraction, observation time, weight, presence of diabetes mellitus (no, yes), presence of hypertension (no, yes), presence of pneumonia (no, yes), presence of chronic kidney disease (CKD) (no, yes), presence of anemia (no, yes) and New York Heart Association (NYHA) class (class II, class III, class IV). NYHA functional classification was used for classification of severity of symptoms. The classes (I-IV) are:- Class I: cardiac disease, but no symptoms and no limitation in ordinary physical activity; Class II: mild symptoms (mild shortness of breath and/or angina) and slight limitation during ordinary activity; Class III: Marked limitation in activity due to symptoms, even during less-than-ordinary activity; Class IV: Comfortable only at rest, severe limitations, experiences symptoms even while at rest, mostly bed bound patients.

\section{Data Processing and Analysis}

In this study, both descriptive and inferential statistical analysis were used. The researcher analysis, a separate model for each longitudinal and survival model, and a joint model of longitudinal and survival analysis for longitudinal measurements and time-to-event outcome of CHF patients linked by subject-specific random effects. Data management has been done by SAS 9.4 and $\mathrm{R}$ version 4.0.2 statistical software. SPSS version 23.0 has been used for data entering. Statistical decision was made at $5 \%$ level of significance.

\section{Ethical Considerations}

The data used in the current investigation was collected previously by the health staff for treatment purpose/diagnosis of heart failure and to start follow-up treatment. To use this previously collected data, an ethical approval certificate had been obtained from University of Gondar Ethical Approval Committee, University of Gondar, Ethiopia, with reference number: RCS/381/2019. The informed consent was waived due to the anonymized data, and this study was conducted in accordance with the Declaration of Helsinki in which it is 
stated that in medical research using identifiable human material or data, physicians must normally seek consent for the collection, analysis, storage and/or reuse.

\section{Results}

\section{Descriptive Statistics}

Descriptive statistics were used to summarize the characteristics of participants. The respondent's characteristics are summarized in Table 1 . Out of the total of 302 patients,
168 (55.6\%) were females and the remaining 134 (44.4\%) were males. More than half of the CHF patients 213 $(70.5 \%)$ were rural residents. For all the CHF patients, 118 (39.1\%) had biventricular heart failure, 84 (27.8\%) had faced left-sided heart failure, and the remaining 100 $(33.1 \%)$ had faced right-sided heart failure. For the reason that HF is not a disease by itself, patients with HF have other causes, out of this cause of heart failure for patients, $96(31.8 \%)$ were by VHD, 42 (13.9\%) were by HHD, 26

Table I Results of Descriptive Measures of Categorical Variables of CHF Patient's Data Taken at FHRH from 2016-2018

\begin{tabular}{|c|c|c|c|c|}
\hline Characteristics & Category & No. of Patients (\%) & Observed Events (\%) & Mean of PR \\
\hline \multirow[t]{2}{*}{ Gender } & Female & $168(55.6)$ & $29(17.3)$ & 87.25 \\
\hline & Male & $134(44.4)$ & $74(55.2)$ & 90.20 \\
\hline \multirow[t]{2}{*}{ Residence } & Rural & $213(70.5)$ & $82(38.5)$ & 88.58 \\
\hline & Urban & $89(29.5)$ & $21(23.6)$ & 88.15 \\
\hline \multirow[t]{3}{*}{ Type of CHF of patients } & Left side failure & $84(27.8)$ & $30(35.7)$ & 87.42 \\
\hline & Right side failure & $100(33.1)$ & $37(37.0)$ & 89.46 \\
\hline & Biventricular failure & $118(39.1)$ & $36(30.5)$ & 88.40 \\
\hline \multirow[t]{6}{*}{ Etiology of heart failure } & VHD & $96(31.8)$ & $3 I(32.3)$ & 87.27 \\
\hline & HHD & $42(13.9)$ & $13(31.0)$ & 86.44 \\
\hline & IHD & $26(8.6)$ & $8(30.8)$ & 91.87 \\
\hline & Cor-pulmonale & $35(11.6)$ & $14(40)$ & 91.24 \\
\hline & Dilated Cardiomyopathy & $66(21.9)$ & $22(33.3)$ & 88.68 \\
\hline & Other etiology's & $37(12.3)$ & $15(40.5)$ & 88.46 \\
\hline \multirow[t]{3}{*}{ NYHA class } & Class II & $50(16.6)$ & $7(14.0)$ & 86.43 \\
\hline & Class III & $106(35.1)$ & $22(20.8)$ & 87.26 \\
\hline & Class IV & I 46 (48.3) & $74(50.7)$ & 90.00 \\
\hline \multirow[t]{2}{*}{ Diabetes Mellitus } & No & $266(88.1)$ & $94(35.3)$ & 88.50 \\
\hline & Yes & $36(11.9)$ & $9(25.0)$ & 88.10 \\
\hline \multirow[t]{2}{*}{ Hypertension } & No & $229(75.8)$ & $56(24.5)$ & 88.30 \\
\hline & Yes & $73(24.2)$ & $47(64.4)$ & 88.99 \\
\hline \multirow[t]{2}{*}{ Pneumonia } & No & $231(76.5)$ & $63(27.3)$ & 87.82 \\
\hline & Yes & $71(23.5)$ & $40(56.3)$ & 90.50 \\
\hline \multirow[t]{2}{*}{ Chronic Kidney Disease } & No & $256(84.8)$ & $80(31.3)$ & 88.48 \\
\hline & Yes & $46(15.2)$ & $23(50.0)$ & 88.25 \\
\hline \multirow[t]{2}{*}{ Anemia } & No & $239(79.1)$ & $75(31.4)$ & 88.08 \\
\hline & Yes & $63(20.9)$ & $28(44.4)$ & 89.75 \\
\hline \multirow[t]{2}{*}{ Censoring status } & Censored & $199(65.9)$ & & \\
\hline & Event (default) & $103(34.1)$ & & \\
\hline Mean age (Std.dev) in year & $47.88(19.116)$ & & & \\
\hline Mean weight (Std.dev) in kilogram & $56.95(8.239)$ & & & \\
\hline Mean LVEF (Std.dev) in \% & $51.12(15.02)$ & & & \\
\hline
\end{tabular}

Abbreviations: CHF, congestive heart failure; HHD, hypertensive heart disease; IHD, ischemic heart disease; LVEF, left ventricular ejection fraction; VHD, valvular heart disease. 
$(8.6 \%)$ were by IHD, 35 (11.6\%) were by cor-pulmonale, $66(21.9 \%)$ were by dilated cardiomyopathy and the remaining 37 (12.3\%) patients were by other etiologies. When we observed the NYHA class of CHF patients, 50 $(16.6 \%)$ of them were NYHA class II, 106 (35.1\%) were class III, 146 (48.3\%) were class IV. Furthermore, from the comorbidities of heart failure patients, 73 (24.2\%) were suffering from hypertension, 36 (11.9\%) from diabetes mellitus, $71(23.5 \%)$ from pneumonia, 46 (15.2\%) from coronary kidney disease and $63(20.9 \%)$ had anemia.

Out of the total $302 \mathrm{CHF}$ treatment followers, about $55.2 \%$ male respondents defaulted from treatment and the remaining were censored. On the other hand, about 17.3\% female respondents defaulted and the remaining respondents were censored. Based on the patient's residence, out of 213 rural and 89 urban patients, $38.55 \%$ and $23.6 \%$ of the respondents had an occurrence of an event, respectively. When we saw NYHA class of CHF patients, NYHA class II, $14.0 \%$, NYHA class III, 20.8\%, and NYHA class IV, $50.7 \%$ of respondents were at default. The mean pulse rate of female and male patients was 87.25 and 90.20 , respectively. The remaining variables are described in the same way.

The mean of baseline age, weight, and left ventricular ejection fraction of patients were 47.88 years (with a standard deviation of 19.116 years), 56.95 kilograms (with a standard deviation of 8.239 kilograms) and $51.12 \%$ (with a standard deviation of $15.02 \%$ ), which means that averagely $51.12 \%$ of blood in the left ventricle is pushed out with each heartbeat respectively (see Table 1).

\section{Exploratory Data Analysis}

Exploratory data analysis was conducted in order to investigate various associations, structures and patterns exhibited in the data set. In addition, the individual profile plots and mean structure plots were obtained in order to gain some insights of the data. To understand the association between repeat measured pulse rate and observation time, individual profile plots were employed. Individual profiles demonstrate the variability (within and between patients) in pulse rate counts per minute of heart failure patient's. It indicates that the need for random intercept, patients started differently at a different baseline pulse rate. In addition to random intercepts, there is also a need for random slopes to allow subjects to have different slopes and account for the instability between subject variability at different time points. To explore the mean change of pulse rate measure over time LOESS smoothing techniques over individual profile plots was used since we have unbalanced longitudinal data. The bold red line LOESS smoothing technique suggests that the mean structure of the variable is nearly linear (ie, the relationship between pulse rate and time seems to be linear). The plotted profiles tend to generate a linear decreasing pattern, which rationalizes the use of linear mixed effects model to analyze the trajectory of pulse rate (Figure 1).

Similarly, we explore the mean plot of pulse rate over time to describe the longitudinal change in pulse rate of CHF patients. The figure revealed that there seems a slight decrease in average pulse rate over time (Figure 2).

\section{Separate Analysis of Longitudinal Data}

As can be observed from Table 2 below, the analysis of the longitudinal data was based on linear mixed effects model incorporates patient-specific pulse rate variability. In this model, among the six predictors included in the model age, gender, left ventricular ejection fraction (LVEF), NYHA Class IV, and follow-up time were statistically significant. The random effect part of the above table indicates how the evolution of pulse rate for the $i$ th subjects deviates from the average evolution in the population. The results showed that the variance and covariance of the random effects are significantly different from zero. This indicates that the pulse rate values at baseline vary across subjects and the change of pulse rate measured over time varies between subjects (see Table 2).

\section{Separate Analysis of Survival Data}

Among the candidate variables considered for building multivariable Cox, the stepwise procedure picked up six variables, weight, gender, NYHA class, presence of hypertension, presence of pneumonia, and presence of coronary kidney disease. The proportional hazards assumption asserts that the hazard ratios are constant over time. That means the risk of failure must be the same no matter how long the subjects have been followed. In order to test this assumption global tests were used.

The results of the separate survival models are presented in Table 3. In this separate analysis of the Cox regression model, the gender of CHF patients, NYHA class (IV) patients, patients who had hypertension, pneumonia, and CKD were positively associated with defaulting time. However, the weight of patients was negatively 


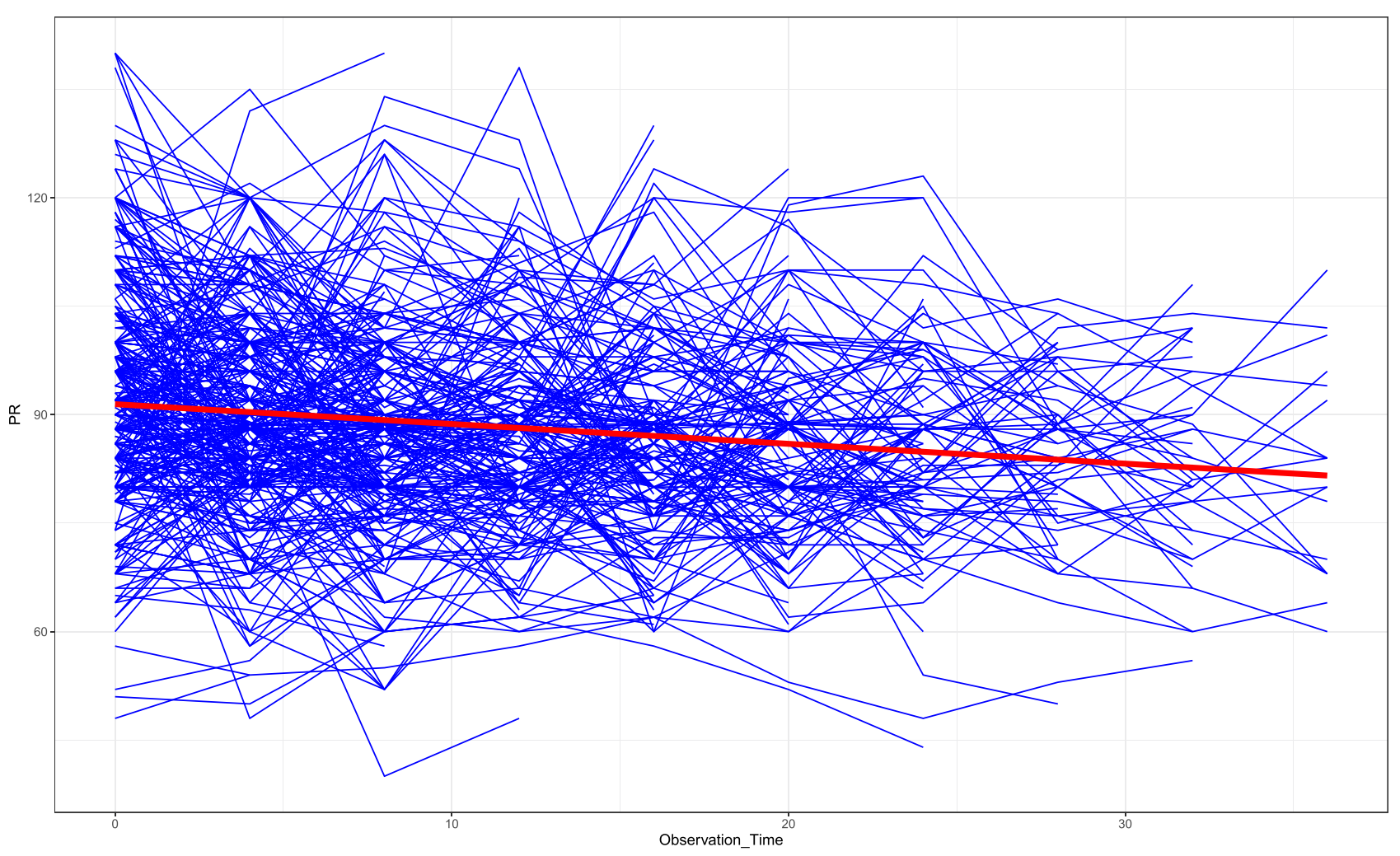

Figure I Individual profiles plot with average trend line for CHF patients under follow up.

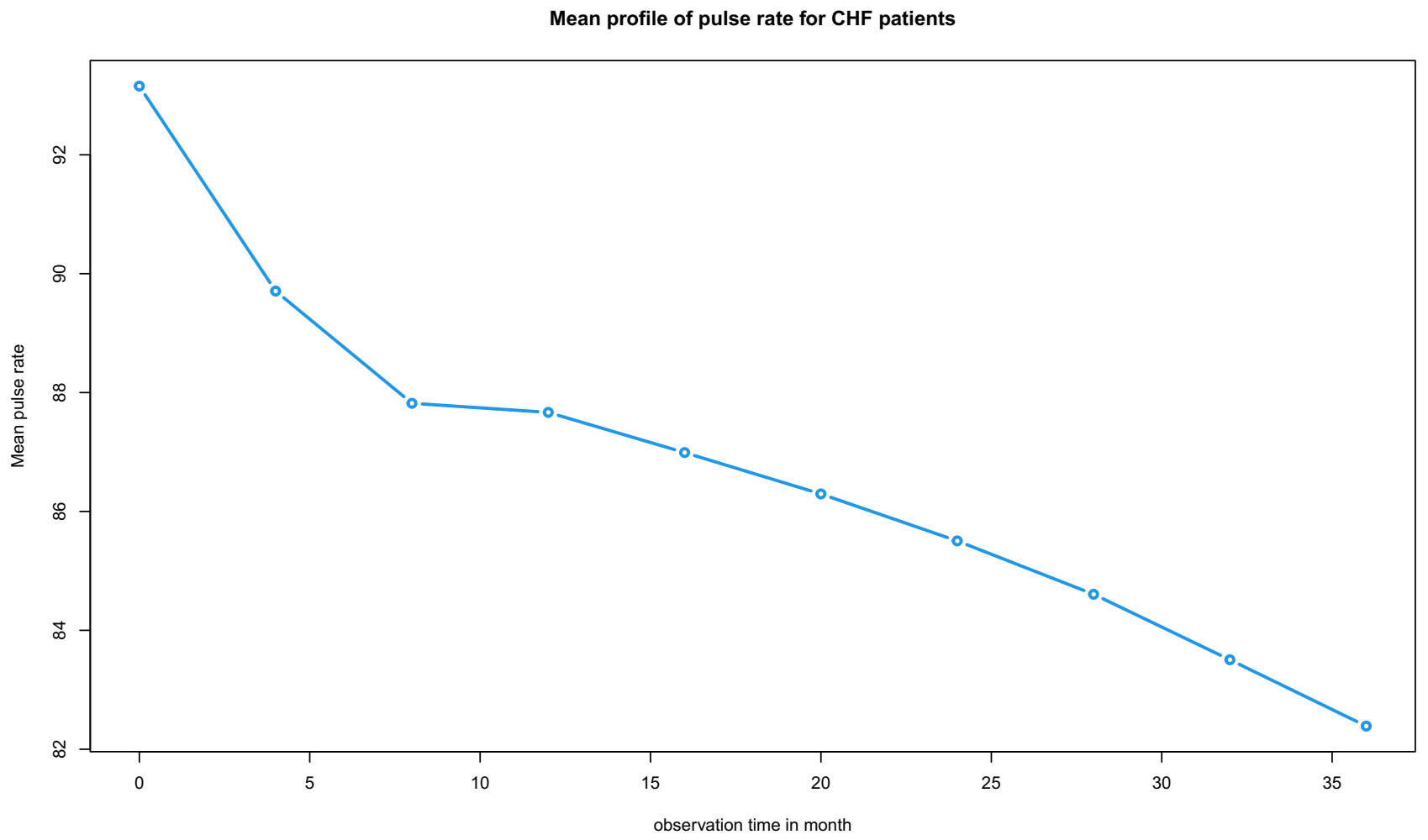

Figure 2 Mean profile plot for CHF patients under follow up. 
Table 2 Parameter Estimation of Linear Mixed Model for CHF Patient's Data Taken at FHRH from 2016-2018, with (UN) Covariance Structure

\begin{tabular}{|c|c|c|c|c|}
\hline Parameter & Estimate & SE & $95 \% \mathrm{Cl}$ & p-value \\
\hline Intercept & 97.4476 & 3.2803 & $91.0126,103.8827$ & $<0.0001$ \\
\hline Observation (follow up) time & -0.2761 & 0.0422 & $-0.3588,-0.1933$ & $<0.0001$ \\
\hline Age & -0.0842 & 0.0317 & $-0.1464,-0.0221$ & 0.0080 \\
\hline \multicolumn{5}{|l|}{ Gender (ref. $=$ female) } \\
\hline Male & 2.4439 & 1.2003 & $0.0893,4.7985$ & 0.0419 \\
\hline \multicolumn{5}{|l|}{ Pneumonia(ref.=no) } \\
\hline Yes & 2.6245 & 1.4010 & $-0.1239,5.3728$ & 0.0612 \\
\hline \multicolumn{5}{|l|}{ NYHA class (ref=class II) } \\
\hline Class III & $1.148 \mid$ & 1.7586 & $-2.3019,4.5981$ & 0.5140 \\
\hline Class IV & 3.3594 & 1.6964 & $0.0315,6.6873$ & 0.0479 \\
\hline LVEF & -0.1095 & 0.0396 & $-0.1872,-0.0318$ & 0.0058 \\
\hline Random effects & Estimates & Std.Err & $95 \% \mathrm{Cl}$ & $\mathrm{p}$-value \\
\hline Intercept $\left(\operatorname{Std} v\left(b_{0 i}\right)\right)$ & 10.1769 & 0.0656 & $8.9490,11.5732$ & $<0.0001$ \\
\hline Observation time $\left(\operatorname{Stdv}\left(b_{1 i}\right)\right)$ & 0.3538 & 0.1676 & $0.2548,0.4914$ & 0.0174 \\
\hline $\operatorname{cor}\left(b_{0 i}, b_{1 i}\right)$ & -0.5261 & 0.2813 & $-0.6944,-0.3031$ & 0.0307 \\
\hline Residual & 10.9695 & 0.0209 & $10.5289,11.4286$ & $<0.0001$ \\
\hline
\end{tabular}

Abbreviation: ref, reference category for categorical variables.

associated with defaulting time for treatment of CHF patients at a $5 \%$ level of significance (see Table 3).

\section{Joint Model of Survival and Longitudinal Analysis}

In many clinical studies, subjects are followed up repeatedly and response data collected, and this response data may be censored by time-to-event outcome. A common objective in follow-up studies is to characterize the relationship between longitudinal measurements and time-to-event outcomes. If the two outcome processes are correlated, modelling the longitudinal and event-time outcomes separately can be inefficient and can lead to biased estimation. To alleviate these problems, we fit the joint model approach for longitudinal (pulse rate measure) and time-to-event (time to default from treatment) data, to identify predictors that affect the two responses and to check whether there is an association between unobserved longitudinal outcome (PR) and time to default of CHF patients. The result of the joint model could be obtained by combining the selected random-intercept-and -slope model and Cox-proportional hazard model.
Table 4 displays the results of joint modeling for the longitudinal and survival process, the result shows that the predictors age, LVEF, gender, NYHA class, and observation time were significantly associated with average PR. In addition, the result shows that the predictors gender, hypertension, CKD, pneumonia, and NYHA class were significantly associated with time to default of CHF patients in the survival sub model. The estimate of the association parameter $(\alpha)$ is positive (0.0307), indicating that pulse rate is positively associated with the risk of default of patients to heart failure treatment. This means that a decreasing trend in the pulse rate of patients undergoing heart failure treatment significantly reduces the risk of defaulting for those patients. Moreover, the estimate of the association parameter in the joint analysis is significantly different from zero, providing strong evidence of association between the effects of the longitudinal outcome on the risk of an event. We also observed that the random effect parameter estimates for joint model analysis. It shows that the variation of the random intercepts was higher than the random slope, which implies a higher baseline difference. The output also displays the correlation 
Table 3 Results for Predictors of Separate Survival Model Time to Default from Treatment of CHF Patients with FHRH from 20I6-20I8

\begin{tabular}{|c|c|c|c|c|}
\hline Parameters & Estimates & SE & HR (95\% Cl) & p-value \\
\hline \multicolumn{5}{|l|}{ Gender (ref.=Female) } \\
\hline Male & 1.3474 & 0.2229 & $3.8474(2.4854,5.9556)$ & $<0.000 I^{* *}$ \\
\hline Baseline Weight & -0.0327 & 0.0117 & $0.9679(0.9460,0.9903)$ & $0.005 I^{* *}$ \\
\hline \multicolumn{5}{|l|}{ Hypertension (ref.=no) } \\
\hline Yes & 0.96902 & 0.20585 & $2.6354(1.7604,3.945 I)$ & $<0.0001^{* *}$ \\
\hline \multicolumn{5}{|l|}{ CKD (ref.=no) } \\
\hline Yes & 0.63992 & 0.24216 & I.8963 (I.I797,3.0482) & $0.00823 * *$ \\
\hline \multicolumn{5}{|l|}{ NYHA Class (ref. = Class II) } \\
\hline Class III & 0.47794 & 0.43873 & $1.6128(0.6825,3.8108)$ & 0.27598 \\
\hline Class IV & 1.12101 & 0.40050 & $3.068(1.3994,6.7260)$ & $0.00513 * *$ \\
\hline \multicolumn{5}{|l|}{ Pneumonia (ref.=no) } \\
\hline Yes & 0.53431 & 0.20546 & I.7063 (I.I407,2.5523) & $0.00931 * *$ \\
\hline
\end{tabular}

Note: **Indicates the significance of covariates at $5 \%$ level of significance.

coefficient between the random intercept and the random slope, which indicates that the slope of time and intercept are dependent because of the dependence of the slope and the intercept of each individual. Therefore, the variability between patients in intercept was 100.2722 , the variability between patients in slope was 0.1219 , the correlation between intercepts and slope were -0.5468 , which indicates that there is a negative correlation between the intercept and slope of linear time effect for the random part and the variability within patients were 120.7801 (see Table 4).

\section{Comparison of Separate and Joint Models}

In both the longitudinal submodel and separate longitudinal model follow-up times, age, gender, LVEF, and NYHA class IV were statistically significant predictors. However, the longitudinal submodel has a narrow confidence interval, which indicates its standard error is small for all significant predictors compared to the separate models. In the survival sub-model of the joint model, except weight, all predictors included in the model were significantly associated with the hazard of defaulting. When evaluating the overall performance of both the separate and joint models in terms of model parsimony and goodness of fit, the joint model was preferred as it has a smaller total AIC than the separate model. Moreover, the statistical significance of the association parameter is evidence that the joint model is better than the separate models. ${ }^{14}$ As (Table 4) revealed, the estimate of the association parameter in the survival sub-model analysis under the joint model was significantly different from zero, this indicates that the two outcomes were correlated. Therefore, the joint model was found preferable to fit the data better over the separate one.

\section{Discussion}

In this study, three different models were explored, the linear mixed effects model, a Cox proportional hazard model for each outcome independently, and joint modeling of the two outcomes together (ie longitudinal and survival). Since joint model building usually starts from separate models for each component, initially each data was analyzed separately. Such a separate analysis is preferred for several reasons. Firstly, it helps to specify the mean response of the model. Secondly, the random effects to be included in the longitudinal model can be easily determined, and thirdly, the initial values to be provided for the joint models can be obtained.

After the most suitable separate models had been decided for the data, the proposed joint models were applied to the data, with the aim of investigating the effects of repeat measured pulse rate measurements on time-to-default. In the longitudinal sub-model age, left ventricular ejection fraction, follow-up time, gender, and patients with NYHA class IV are statistically associated with the progression of pulse rate. An increment in the progression of pulse rate over follow-up time indicates the severity of the disease, whereas the decrease in the evolution of the longitudinal measured pulse rate indicates the 


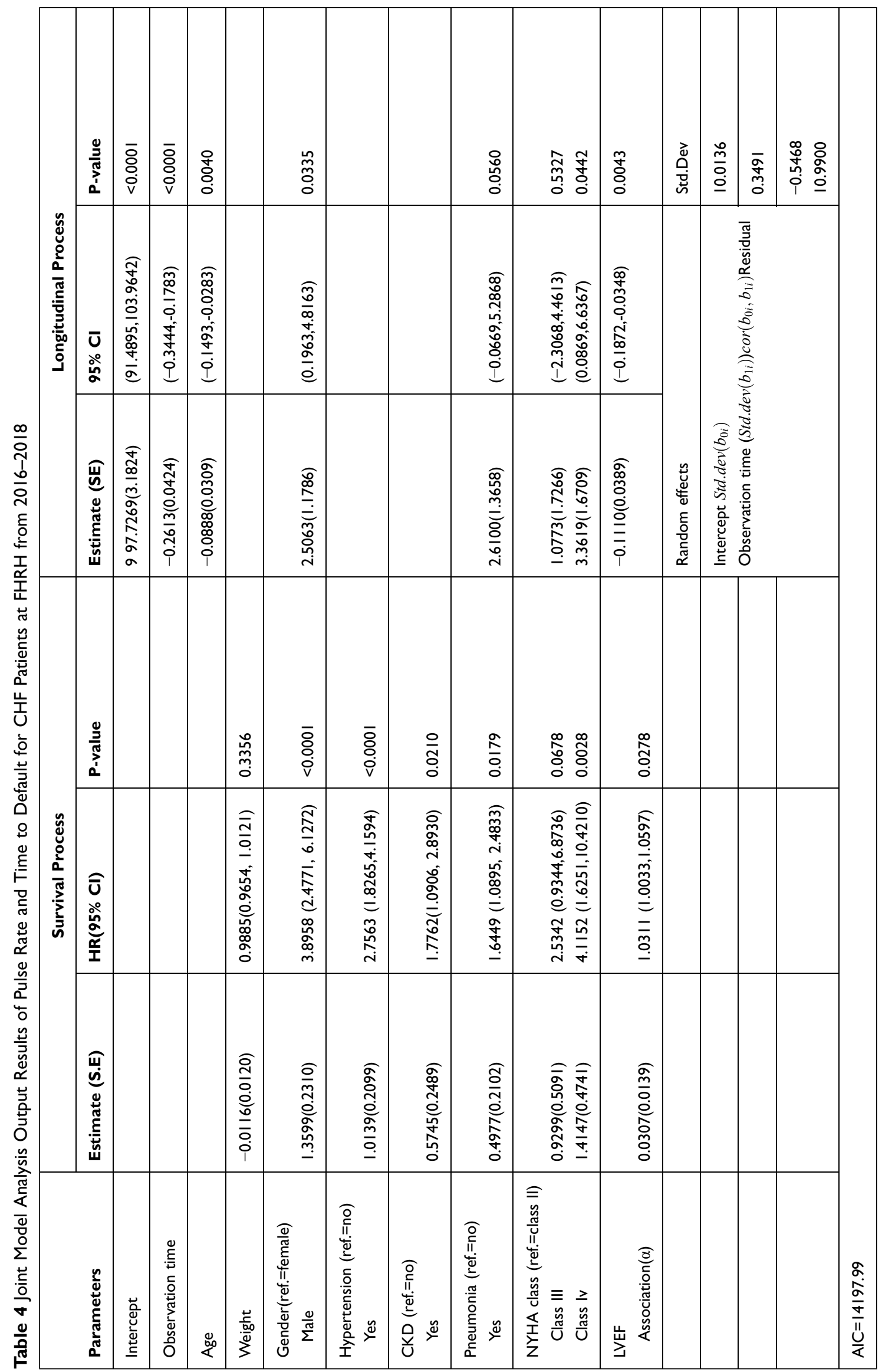


effectiveness of the treatment (reduction in severity of the disease). In this study, follow-up time had a significant negative effect on the average evolution of pulse rate. Patients with more follow-up time made a particular decrease in pulse rate $(\mathrm{P}<0.0001)$. Which means a follow-up time in months reduces the severity of the disease.

When the age of a patient increases by one year, the average pulse rate of patients is decreased by $8.88 \%$, while all other predictors are held constant $(\mathrm{P}=0.0040)$. This finding is in line with other studies, ${ }^{7,11,16}$ which showed that ages were found to be negatively associated with the average evolution of PR.

When the left ventricle ejection fraction of patients is increased by one unit, the average pulse rates of patients are decreased by $11.10 \%$, while all other predictors are held constant $(P=0.0043)$. This means that if pushed out blood in the left ventricle in each heart beat increased by one percent, the average pulse rate of patients deceased by $11.10 \%$. This finding is in line with other studies, ${ }^{7,11}$ which showed that LVEF were found to be negatively associated with the average evolution of pulse rate. However, our finding contradicts the earlier study by Nesbitt et $\mathrm{al}^{17}$ which shows no evidence of an association between left ventricular ejection fraction and quality of life of HF patients.

Among New York Heart Association classes (NYHA class), NYHA class IV patients were found to have a significant difference compared to NYHA class II patients $(\mathrm{P}=0.0442)$. This implies that the progression of the average pulse rate of NYHA class IV patients starting CHF treatment were higher by 3.3619 than those NYHA class II patients. This finding is in line with other studies, ${ }^{7}$ which showed that NYHA class was positively significantly associated with PR. Moreover, this finding is in line with the previous finding by Nesbitt et al ${ }^{17}$ which shows that a higher NYHA class reduced the quality of life of CHF patients.

The average pulse rate of male patients is greater by 2.5063 compared to female patients, while other covariates are kept constant $(\mathrm{P}=0.0335)$. This result also conforms to the result of Nesbitt et al ${ }^{17}$ which shows that male gender is associated with reduced quality of life of CHF patients. However, our study contradicts earlier studies, ${ }^{7,16}$ which showed that female patients had a higher pulse rate than males during the followup. However, in our finding male patients have a higher significant effect in the evolution of the average pulse rate of patients compared to female patients.

In the survival submodel, male gender, hypertension, CKD, pneumonia, and NYHA class IV are significantly associated with the hazard of defaulting from treatment.
Male patients were associated with a risk of default $(\mathrm{HR}=$ 3.8958, 95\% CI: 2.4771, 6.1272, $\mathrm{P}=<0.0001$ ) that was a 3.8958 times higher risk of default compared to female patients holding other covariates in the model constant. This result was supported by most other studies, ${ }^{18}$ which showed female patients had a slightly higher survival probability than male patients. This study was also in line with the studies ${ }^{19} 20$ which indicates that male gender had a significant effect on mortality among heart failure patients. However, our study contradicts with an earlier study, $^{21}$ which showed no significant differences in mortality among gender of heart failure patients.

The estimated risk of defaulting for a patient with NYHA class (IV) compared to NYHA class (II) patient is $(\mathrm{HR}=4.1152,95 \% \mathrm{CI}: 1.6251,10.4210, \mathrm{P}=0.0028)$, indicating that the hazard rate of defaulting from treatment for NYHA class (IV) patients is around four times higher than NYHA class (II) patients, holding other covariates in the model constant. This result agress with a previous study, ${ }^{19}$ which indicates that higher NYHA class patients had a higher risk of death.

The estimated risk of defaulting for a heart failure patient with the presence of hypertension is ( $\mathrm{HR}=2.7563,95 \% \mathrm{CI}$ : $1.8265,4.1594, \mathrm{P}=<0.0001)$, This indicates that the risk of defaulting for hypertensive patients were 2.7563 times higher compared to non-hypertensive patients keeping other variables constant. This finding is in line with previous findings 226 which showed hypertension was positive and significantly associated with the prevalence of HF. Likewise, estimated risk of defaulting for $\mathrm{CHF}$ patients with presence of pneumonia and $\mathrm{CKD}$ as co morbidities was higher $(\mathrm{HR}=1.6449,95 \% \mathrm{CI}$ : 1.0895, 2.4833, $\mathrm{P}=0.0179),(\mathrm{HR}=1.7762,95 \% \mathrm{CI}: 1.0906$, $2.8930, \mathrm{P}=0.0210$ ), respectively. This indicates that the risks of defaulting for pneumonic and CKD patients were 1.6449 and 1.7762 times the risk of defaulting for a patient who had no pneumonia and $\mathrm{CKD}$, respectively, keeping other variables constant.

Furthermore, the estimated value for the association parameter $(\alpha)$ in the joint model is 0.0307 and the hazard ratio is 1.0311 (with 95\% CI: 1.0033, 1.0597) and statistically significant $(p$-value $=0.0278)$. This indicates that there is strong evidence of association between the effects of the longitudinal biomarker to the risk of default. For a unit change in the true value of the repeated measure pulse rate, the relative change in the risk of defaulting is 1.0311 while other variables are kept constant. The results indicate that lower values of pulse rate are associated with better survival, which is in line with the findings, ${ }^{11,16,23}$ higher resting heart rate was predictive of 
earlier mortality and heart rate reduction is associated with improved clinical outcomes in heart failure patients, which is in line with the findings, ${ }^{11,16,23}$ higher resting heart rate is predictive of earlier mortality and heart rate reduction is associated with improved clinical outcomes in heart failure patients. This finding contrasts with the previous finding ${ }^{24}$ which showed that a joint effect of both the mean number of abnormal heart rate (pulse rate) measurements and the risk of re-hospitalization were not statistically significant $(\mathrm{p}=0.1650)$.

\section{Limitation of the Study}

A limitation of the study is that limited secondary data were used. Consequently, important variables such as body mass index, alcoholism, marital status, education level, and smoking status are not available in the patients' charts; we have not used such variables in our research.

\section{Conclusion}

The study investigated and identified factors that are associated with the longitudinal measure PR and defaulting time of $\mathrm{CHF}$ patients from treatment in Felege-Hiwot Referral Hospital using both separate and joint model analysis. For this specific study, the joint model was the better fit than the separate survival and longitudinal models. Longitudinal sub-models showed that: age, LVEF, follow-up time of the patients, gender, and NYHA class were significant predictors of the progression change of pulse rate. Since follow-up time of patients had a negative impact, we conclude that the rate of progression of pulse rate decreases over time. Moreover, the survival submodel showed that CKD, hypertension, pneumonia, NYHA class, male gender, and unobserved true PR were positively associated with the time to default. When evaluating the overall performance of both the separate and joint models in terms of model parsimony, goodness of fit, smaller total AIC, and the statistical significance of association parameters, the joint model performs better. Thus, we concluded that the joint model was preferred for simultaneous analyses of repeated measurement and survival data.

\section{Abbreviations}

AIC, Akaike's information criterion; $\mathrm{CHF}$, chronic or congestive heart failure; CI, confidence interval; CKD, chronic kidney disease; FHRH, Felege-Hiwot Referral Hospital; HR, hazard ratio; HHD, hypertensive heart disease, IHD, ischemic heart disease; LVEF, left ventricular ejection fraction; NYHA, New York Heart Association; PR, pulse rate; SPSS, statistical package for social science;
Std.Dev, standard deviation; SE, standard error; SSA, SubSaharan Africa; VHD, valvular heart disease; WHO, World Health Organization.

\section{Data Sharing Statement}

The authors confirmed that the data used for this research is available from the corresponding author.

\section{Ethical Approval and Consent to Participate}

The data used in the current investigation was collected previously by the health staff for treatment purpose/diagnosis of heart failure and to start follow-up treatment. To use this previously collected data, an ethical approval certificate had been obtained from University of Gondar Ethical Approval Committee, University of Gondar, Ethiopia, with reference number: RCS/381/2019. The informed consent was waived due to the anonymized data, and this study was conducted in accordance with the Declaration of Helsinki in which it is stated that in medical research using identifiable human material or data, physicians must normally seek consent for the collection, analysis, storage and/or reuse.

\section{Consent for Publication}

This manuscript has not been published elsewhere and is not under consideration by any other journal. All authors approved the final manuscript and agreed to its submission. We agreed about the authorship and the order of authors for this manuscript.

\section{Acknowledgments}

We would like to acknowledge University of Gondar for their financial support. We would also like to express our special thanks to Doctor Getaneh Kassie and Felege-Hiwot Referral Hospital staff members for providing important information and unreserved support. We extend our special thanks to all data collectors and supervisors.

\section{Author Contributions}

All authors made substantial contributions to conception and design, acquisition of data, or analysis and interpretation of data., whether that is in the conception, study design, execution, acquisition of data, analysis and interpretation, or in all these areas; took part in drafting, revising or critically reviewing the article; gave final approval of the version to be published; have agreed on the journal to which the article has been 
submitted; and agree to be accountable for all aspects of the work.

\section{Funding}

There is no funding to report.

\section{Disclosure}

The authors report no financial or nonfinancial conflicts of interest for this work.

\section{References}

1. Ziaeian B, Fonarow GC. Epidemiology and aetiology of heart failure. Nat Rev Cardiol. 2016;13(6):368-378. doi:10.1038/nrcardio.2016.25

2. Cook C, Cole G, Asaris P, et al. 49 The annual global economic burden of heart failure. Heart. 2014;100(Suppl 3):A28-A29. doi:10.1136/heartjnl-2014-306118.49

3. Ntusi NB, Mayosi BM. Epidemiology of heart failure in sub-Saharan Africa. Expert Rev Cardiovasc Ther. 2009;7(2):169-180. doi:10.1586/14779072.7.2.169

4. Agbor VN, Essouma M, Ntusi NAB, et al. Heart failure in sub-Saharan Africa: a contemporaneous systematic review and meta-analysis. Int $J$ Cardiol. 2018;257:207-215. doi:10.1016/j. ijcard.2017.12.048

5. WHO. Global Status Report on Noncommunicable Diseases 2014. World Health Organization; 2014.

6. Zeru MA. Assessment of major causes of heart failure and its pharmacologic management among patients at Felege Hiwot referral hospital in Bahir Dar, Ethiopia. J Public Health Epidemiol. 2018;10 (9):326-331. doi:10.5897/JPHE2018.1046

7. Fissuh YH, Muletav G. A joint model for a longitudinal pulse rate and respiratory rate of congestive heart failure patients: at Ayder Referral Hospital of Mekelle University, Tigray, Ethiopia. J Biom Biostat. 2015;6(5):1. doi:10.4172/2155-6180.1000260

8. Greene SJ, Vaduganathan M, Wilcox JE, et al. The prognostic significance of heart rate in patients hospitalized for heart failure with reduced ejection fraction in sinus rhythm: insights from the EVEREST (Efficacy of Vasopressin Antagonism in Heart Failure: outcome Study With Tolvaptan) trial. JACC Heart Fail. 2013;1 (6):488-496.

9. Aarts LA, Jeanne V, Cleary JP, et al. Non-contact heart rate monitoring utilizing camera photoplethysmography in the neonatal intensive care unit-A pilot study. Early Hum Dev. 2013;89(12):943-948. doi:10.1016/j.earlhumdev.2013.09.016

10. Custodis F, Schirmer SH, Baumhäkel M, et al. Vascular pathophysiology in response to increased heart rate. $J$ Am Coll Cardiol. 2010;56(24):1973-1983. doi:10.1016/j.jacc.2010.09.014
11. Kurgansky KE, Schubert P, Parker R, et al. Association of pulse rate with outcomes in heart failure with reduced ejection fraction: a retrospective cohort study. BMC Cardiovasc Disord. 2020;20 (1):1-11. doi:10.1186/s12872-020-01384-6

12. Farmakis D, Stafylas P, Giamouzis G, Maniadakis N, Parissis J. The Medical and Socioeconomic Burden of Heart Failure: A Comparative Delineation with Cancer. Elsevier; 2016.

13. Wajner A, Zuchinali P, Olsen V, Polanczyk CA, Rohde LE. causes and predictors of in-hospital mortality in patients admitted with or for heart failure at a Tertiary Hospital in Brazil. Arq Bras Cardiol. 2017;109:321-330.

14. Seid A, Getie M, Birlie B, Getachew Y. Joint modeling of longitudinal CD4 cell counts and time-to-default from HAART treatment: a comparison of separate and joint models. Elect J Appl Stat Anal. 2014;7(2):292-314.

15. Grover G, Swain P, Deo V, Varshney M. A joint modeling approach to assess the impact of CD4 cell count on the risk of loss to follow up in HIV/AIDS patients on antiretroviral therapy. Int J Stat Appl. 2015;5(3):99-108.

16. Castagno D, Skali H, Swedberg K, et al. Heart rate and outcomes in a broad spectrum of patients with chronic heart failure: results from the CHARM programme. Heart Fail Congr. 2011:10:50.

17. Nesbitt T, Doctorvaladan S, Southard JA, et al. Correlates of quality of life in rural heart failure patients. Circ Heart Fail. 2014;7:882.

18. Hailay A, Kebede E, Mohammed K. Survival during treatment period of patients with severe heart failure admitted to Intensive Care Unit (ICU) at Gondar University Hospital (GUH), Gondar, Ethiopia. Am J Health Res. 2015;3:257-269.

19. Barlera S, Luigi T, Grazia Franzosi M, et al. Predictors of mortality in 6975 patients with chronic heart failure in the Gruppo Italiano per lo Studio della Streptochinasi nell'Infarto Miocardico-Heart Failure trial: proposal for a nomogram. Circ Heart Fail. 2013;6(1):31-39.

20. Zomer AC, Vaartjes I, van der Velde ET, et al. Heart failure admissions in adults with congenital heart disease; risk factors and prognosis. Int $J$ Cardiol. 2013;168(3):2487-2493. doi:10.1016/j. ijcard.2013.03.003

21. Ahmad T, Munir A, Bhatti SH, et al. Survival analysis of heart failure patients: a case study. PLoS One. 2017;12(7):e0181001. doi:10.1371/ journal.pone. 0181001

22. Beck H, Titze SI, Hübner S, et al. Heart failure in a cohort of patients with chronic kidney disease: the GCKD study. PLoS One. 2015;10 (4):e0122552. doi:10.1371/journal.pone.0122552

23. Kupper N, Denollet J, Widdershoven J, Kop WJ. Cardiovascular reactivity to mental stress and mortality in patients with heart failure. JACC Heart Fail. 2015;3(5):373-382.

24. Njagi EN. Joint Models for Survival and Longitudinal Data, Missing Data, and Sensitivity Analysis, with Applications in Medical Research [Doctoral dissertation, PhD Thesis]. London School of Hygiene and Tropical Medicine; 2013
Research Reports in Clinical Cardiology

\section{Publish your work in this journal}

Research Reports in Clinical Cardiology is an international, peerreviewed, open access journal publishing original research, reports, editorials, reviews and commentaries on all areas of cardiology in the clinic and laboratory. The manuscript management system is completely online and includes a very quick and fair peer-review system. Visit http://www.dovepress.com/testimonials.php to read real quotes from published authors. 